\title{
QUASICONVEXIFICATION IN 3-D FOR A VARIATIONAL REFORMULATION OF AN OPTIMAL DESIGN PROBLEM IN CONDUCTIVITY
}

\author{
Faustino Maestre $^{*} \quad$ Pablo Pedregal ${ }^{\dagger}$ \\ Universidad de Castilla-La Mancha, E.T.S.I. Industriales, 13071, \\ Ciudad Real, Spain
}

\begin{abstract}
We analyze a typical 3-D conductivity problem which consists in seeking the optimal layout of two materials in a given design domain $\Omega \subset \mathbb{R}^{3}$ by minimizing the $L^{2}$-norm of the electric field under a constraint on the amount on each material that we can use. We utilize a characterization of the three-dimensional divergence-free vector fields which is especially appropriate for a variational reformulation. By using gradient Young measures as a main tool, we can give an explicit form of the "constrained quasiconvexification" of the cost density. This result is similar to the one in the 2-D situation. However, the characterization of the divergence-free vector fields introduces a certain nonlinearity in the problem that needs to be addressed properly.
\end{abstract}

\section{Introduction}

In this paper, we will study a typical optimal design problem in conductivity, which consists in looking for the optimal distribution of two different conducting materials with isotropic constants $\alpha$ and $\beta(0<\alpha<\beta)$ on a domain $\Omega \subset \mathbb{R}^{3}$, such that it minimizes a certain functional cost which depends on the underlying electric field of the state equation in the form

$$
I(\chi)=\int_{\Omega} a(x, \chi(x))|\nabla u(x)-F(x)|^{2} d x
$$

where $u$ is the unique solution of

*e-mail:Faustino.Maestre@uclm.es

${ }^{\dagger}$ e-mail:Pablo.Pedregal@uclm.es 


$$
\begin{array}{rll}
-\operatorname{div}((\alpha \chi+\beta(1-\chi)) \nabla u)=g & \text { in } & \Omega, \\
u=u_{0} & \text { on } & \partial \Omega,
\end{array}
$$

and the functions $a, F, g$ and $u_{0}$ are known. The function $\chi \in L^{\infty}(\Omega,\{0,1\})$ is the design variable and it indicates where we place the $\alpha$-material. The amount of $\alpha$-material is given, and therefore we have to enforce the volume constraint

$$
\int_{\Omega} \chi(x) d x \leq t_{0}|\Omega|
$$

with $t_{0} \in(0,1)$ fixed.

In short form, the optimal design problem is to

$$
\min _{\chi} I(\chi)
$$

under

$$
\begin{array}{rll}
-\operatorname{div}((\alpha \chi+\beta(1-\chi)) \nabla u)=g & \text { in } & \Omega, \\
u=u_{0} & \text { on } & \partial \Omega,
\end{array}
$$

where the admissible set of $\chi$ 's is the set of the characteristic functions over $\Omega$ under the volume constraint

$$
\frac{1}{|\Omega|} \int_{\Omega} \chi(x) d x \leq t_{0} .
$$

This problem has been extensively studied in the two-dimensional case $([1],[2],[10],[13])$, and it has also been examined in the three-dimensional case $([3])$. In this paper, we would like to pursue a more direct analysis of the three-dimensional situation in order to deal with a more natural generalization, as compared to the treatment in ([3]), of the two-dimensional case. In doing so, we will have to overcome a certain non-linear structure which does not have a parallelism in the 2 -D case. This is the result of using Lemma 1 below, a suitable characterization of three-dimensional divergencefree vector fields.

It is well understood the lack of classical solutions for this type of problems. This is the reason why we reformulate the problem through relaxation techniques. The theory of homogenization is an important tool which introduces new types of composites as structural elements through the concepts of H-convergence or G-convergence ([1]). The theory of homogenization is especially useful when we work with non-explicit dependence on the flux $\nabla u(x)$.

Our strategy is directed towards the understanding and computation of the constrained quasiconvexification of a certain integrand which is obtained as a result of a suitable variational reformulation of the problem ([10], [12], [13], [14]). 
Notice, to begin with, that

$$
a|\nabla u-F|^{2}=a|\nabla u|^{2}-2 a \nabla u \cdot F+a|F|^{2},
$$

and that the second part $-2 a \nabla u \cdot F+a|F|^{2}$ is linear in $\nabla u$, therefore it suffices to study the case $F \equiv 0$. The optimal design problem we will treat will be

$$
\min _{\chi \in L^{\infty}(\Omega,\{0,1\})} I(\chi)=\int_{\Omega} a(x, \chi(x))|\nabla u(x)|^{2} d x
$$

subject to,

$$
\begin{array}{rll}
-\operatorname{div}((\alpha \chi+\beta(1-\chi)) \nabla u)=g & \text { in } & \Omega \\
u=u_{0} & \text { on } & \partial \Omega \\
\int_{\Omega} \chi(x) d x \leq t_{0}|\Omega| . & &
\end{array}
$$

We want to reformulate this problem in a different form. We take $G \in H^{1}(\Omega)$ such that

$$
\operatorname{div}(G)=g \text { in } \Omega,
$$

so that the state equation can be rewritten as

$$
-\operatorname{div}((\alpha \chi+\beta(1-\chi)) \nabla u+G(x))=0 \text { in } \Omega \subset \mathbb{R}^{3} .
$$

The treatment of this equation is the main novelty with respect to the two-dimensional case in which the divergence-free fields are characterized as the counterclockwise $\pi / 2$-rotation of the gradients of scalar functions, while in the three-dimensional case the situation is more complex, we will use a characterization by "Clebsch potentials". There are different results in which n-dimensional divergence-free vector fields can been represented in terms of (n-1) arbitrary functions. For the three dimensional case, if $F \in \mathbb{R}^{3}$ with $\operatorname{div}(F)=0$, then there exist Clebsch potentials $v, w$ such that $F=\nabla v \times \nabla w$. The proof of this result is beyond the scope of the present paper and we refer [8], [11]. [18], [15]. In fact, looking at the specialized literature, is seems that this representation en terms of the Clebsch potentials is not always valid ([7]). But this is a fine point for experts which we have avoided. We simply use this representation in the sequel.

Therefore using the Clebsch potentials, we have that (4) can be replaced by,

$$
(\alpha \chi(x)+\beta(1-\chi(x))) \nabla u(x)-\nabla v(x) \times \nabla w(x)+G(x)=0 \quad \text { in } \Omega .
$$

We can therefore use $(u, v, w)$ as new design variables provided they satisfy the following pointwise constraint,

$$
\begin{aligned}
& \alpha \nabla u(x)-\nabla v(x) \times \nabla w(x)+G(x)=0 \\
& \beta \nabla u(x)-\nabla v(x) \times \nabla w(x)+G(x)=0
\end{aligned} \quad \text { a.e. } x \in \Omega .
$$


It's clear that we can identify the design variable $\chi$ with the vector $(u, v, w)$, and conversely a vector $(u, v, w)$ which verify (5) with a design variable $\chi$. We consider then the new design variable $U=\left(U^{(1)}, U^{(2)}, U^{(3)}\right)=(u, v, w)$, where $U: \mathbb{R}^{3} \rightarrow \mathbb{R}^{3}$ and $\nabla U(x) \in \mathbb{R}^{3 \times 3}$.

Let $\Lambda_{\gamma, x}$ be the (non-linear) manifold,

$$
\Lambda_{\gamma, x}=\left\{A \in M^{3 \times 3}: \gamma A^{(1)}-A^{(2)} \times A^{(3)}+G(x)=0\right\}
$$

where $A^{(i)}$ is the $i$-th row of the matrix A.

Put $a_{\alpha}(x)=a(x, 1), a_{\beta}(x)=a(x, 0)$ and

$$
h(x)=\beta a_{\alpha}(x)-\alpha a_{\beta}(x),
$$

and set

$$
\begin{gathered}
W(x, A)= \begin{cases}a_{\alpha}(x)\left|A^{(1)}\right|^{2}, & \text { if } A \in \Lambda_{\alpha, x}, \\
a_{\beta}(x)\left|A^{(1)}\right|^{2}, & \text { if } A \in \Lambda_{\beta, x} \backslash \Lambda_{\alpha, x}, \\
+\infty, & \text { else, }\end{cases} \\
V(x, A)= \begin{cases}1, & \text { if } A \in \Lambda_{\alpha, x}, \\
0, & \text { if } A \in \Lambda_{\beta, x} \backslash \Lambda_{\alpha, x}, \\
+\infty, & \text { else. }\end{cases}
\end{gathered}
$$

It is clear that the original optimal design problem is equivalent to the nonconvex vector variational problem

$$
\min _{U} \hat{I}(U)=\int_{\Omega} W(x, \nabla U(x)) d x
$$

subject to

$$
\begin{gathered}
U \in H^{1}(\Omega)^{3}, U^{(1)}=u_{0} \text { on } \partial \Omega \\
\int_{\Omega} V(x, \nabla U(x)) d x \leq t_{0}|\Omega| .
\end{gathered}
$$

$$
\begin{aligned}
& \psi(t, F)=\alpha \beta(t \alpha+(1-t) \beta)\left|F^{(1)}\right|^{2}+ \\
& {\left[((1-t) \alpha+t \beta)\left(\left|F^{(2)} \times F^{(3)}\right|^{2}+|G|^{2}-2\left(F^{(2)} \times F^{(3)}\right) \cdot G\right)\right]+} \\
& {\left[2 \alpha \beta+t(1-t)(\beta-\alpha)^{2}\right]\left(F^{(1)} \cdot G-\operatorname{det} F\right)} \\
& \varphi(t, F)=\left\{\begin{array}{c}
\frac{h(x)}{t \beta(\beta-\alpha)^{2}}\left(\beta^{2}\left|F^{(1)}\right|^{2}+\left|F^{(2)} \times F^{(3)}\right|^{2}+|G|^{2}-2 \beta \operatorname{det} F+\right. \\
\left.2\left(\beta F^{(1)}-F^{(2)} \times F^{(3)}\right) \cdot G\right)+\frac{a_{\beta}(x)}{\beta}\left(\operatorname{det} F-G \cdot F^{(1)}\right) \\
\quad \text { if } h(x) \geq 0, \psi(t, F) \leq 0, \\
\frac{-h(x)}{(1-t) \alpha(\beta-\alpha)^{2}}\left(\alpha^{2}\left|F^{(1)}\right|^{2}+\left|F^{(2)} \times F^{(3)}\right|^{2}+|G|^{2}-2 \alpha \operatorname{det} F+\right. \\
\left.2\left(\alpha F^{(1)}-F^{(2)} \times F^{(3)}\right) \cdot G\right)+\frac{a_{\alpha}(x)}{\alpha}\left(\operatorname{det} F-G \cdot F^{(1)}\right), \\
\text { if } h(x) \leq 0, \psi(t, F) \leq 0, \\
\text { else. }
\end{array}\right.
\end{aligned}
$$


We will show that the variational problem

$$
\min _{(t, U)} \int_{\Omega} \varphi(t(x), \nabla U(x)) d x
$$

subject to

$$
\begin{gathered}
U \in H^{1}(\Omega)^{3}, U^{(1)}=u_{0} \text { on } \partial \Omega, \psi(t(x), \nabla U(x)) \leq 0, \\
0 \leq t(x) \leq 1, \quad \int_{\Omega} t(x) d x \leq t_{0}|\Omega|,
\end{gathered}
$$

is a relaxation of the original optimal design problem, in the sense explained in the next theorem. This result is the main objective of this work.

Theorem 1 This final variational problem is equivalent to ( a relaxation for ) the original optimal design problem ( determined by (1), (2), (3) ) in the sense that

a) the infima of both problems coincide,

b) there are optimal solutions for the relaxed problem,

c) these solutions codify (in the sense of the Young measure) the optimal microstructures of the original optimal design problem.

For the particular case in which we take $a_{\alpha}(x)=a_{\beta}(x)=1$ and $G=0$, the above formulae simplify to

$$
\begin{aligned}
& h(x)=\beta-\alpha, \\
& \psi(t, F)=\alpha \beta(t \alpha+(1-t) \beta)\left|F^{(1)}\right|^{2}+((1-t) \alpha+t \beta)\left|F^{(2)} \times F^{(3)}\right|^{2} \\
& -\left(2 \alpha \beta+t(1-t)(\beta-\alpha)^{2}\right) \operatorname{det} F \\
& \varphi(t, F)= \begin{cases}\frac{1}{t \beta(\beta-\alpha)}\left(\beta^{2}\left|F^{(1)}\right|^{2}+\left|F^{(2)} \times F^{(3)}\right|^{2}-2 \beta \operatorname{det} F\right)+\frac{1}{\beta} \operatorname{det} F \\
+\infty & \text { if } \psi(t, F) \leq 0, \\
\text { else. }\end{cases}
\end{aligned}
$$

The main new contribution here is to understand how the non-linear character of the manifolds $\Lambda_{\gamma, x}$ above does not in fact interfere with the analogous computations for the 2-D situation. This is so because this nonlinearity is intimately connected to the weak continuity of minors.

The work is organized as follows. We begin by studying a relaxation of the original problem which is given by the constrained quasionvexification. Next, we compute explicitly this relaxation by firstly computing a lower bound (polyconvexification), and then seeking a laminate that recovers this polyconvexification thus showing that the bound is optimal. Finally we prove a basic result for the variational reformulation, Lemma 1. 


\section{Relaxation}

We have recast our optimal design problem as a typical variational problem. We see that it is a non-convex vector problem that we are going to analyze by seeking its relaxation. We use Young measures as a main tool in the computation of the suitable density for the relaxed problem. We are going to follow the same plan that in the two-dimensional case ([2],[10], [13]).

Put

$$
\begin{array}{r}
m=\inf \left\{\int_{\Omega} W(x, \nabla U(x)) d x: U \in H^{1}(\Omega)^{3}, U^{(1)}-u_{0} \in H_{0}^{1}(\Omega),\right. \\
\left.\int_{\Omega} V(x, \nabla U(x)) d x=t_{0}|\Omega|\right\} .
\end{array}
$$

We know $([2])$ that

$$
\begin{aligned}
m \geq \bar{m}=\inf \left\{\int_{\Omega} C Q W(x, \nabla U(x), t(x)) d x: U \in H^{1}(\Omega)^{3},\right. \\
\left.\quad U^{(1)}-u_{0} \in H_{0}^{1}(\Omega), 0 \leq t(x) \leq 1, \int_{\Omega} t(x) d x=t_{0}|\Omega|\right\}
\end{aligned}
$$

where $C Q W(x, F, t)$ is defined by,

$$
C Q W(x, F, t)=\inf \left\{\int_{M^{3 \times 3}} W(x, A) d \nu(A): \nu \in \mathcal{A}(F, t)\right\}
$$

with

$$
\begin{array}{r}
\mathcal{A}(F, t)=\left\{\nu: \nu \text { is a homogeneous } H^{1}\right. \text {-Young measure, } \\
\left.F=\int_{M^{3 \times 3}} A d \nu(A), \int_{M^{3 \times 3}} V(x, A) d \nu(A)=t\right\} .
\end{array}
$$

Notice that the previous inequality will be an equality when $\mathrm{W}$ is a Carathodory function with appropriate growth constrains ([14]). However, in our situtation it is still possible to prove this equality despite the fact that $W$ is not a Carathodory function. Let us consider the following minimization problem

$$
\tilde{m}=\inf \left\{\int_{\Omega} \int_{M^{3 \times 3}} W(x, A) d \nu_{x}(A) d x: \nu \in \mathcal{B}\left(u_{0}, t_{0}\right)\right\}
$$

where

$$
\begin{aligned}
& \mathcal{B}\left(u_{0}, t_{0}\right)=\left\{\nu: H^{1} \text {-Young meas., } \operatorname{supp}\left(\nu_{x}\right) \subset \Lambda_{\alpha} \cup \Lambda_{\beta}, \exists U \in H^{1}(\Omega)^{3},\right. \\
& U^{(1)}-u_{0} \in H_{0}^{1}(\Omega), \int_{\Omega} \int_{M^{3 \times 3}} V(x, A) d \nu_{x}(A) d x=t_{0}|\Omega|, \\
& \left.\nabla U(x)=\int_{M^{3 \times 3}} A d \nu_{x}(A)\right\} .
\end{aligned}
$$

We have the following result. 
Theorem 2 The equalities

$$
m=\bar{m}=\tilde{m}
$$

hold. Moreover, for each measure $\nu \in \mathcal{B}\left(u_{0}, t_{0}\right)$ such that $\operatorname{supp}\left(\nu_{x}\right) \subset \Lambda_{\alpha} \cup \Lambda_{\beta}$ a.e. $x \in \Omega$, there exists a sequence $\left\{\nabla U_{k}\right\}$ such that,

i) $\quad U_{k} \in\left(H^{1}(\Omega)\right)^{3}, U_{k}^{(1)}-u_{0} \in H_{0}^{1}(\Omega),\left\{\left|\nabla U_{k}\right|^{2}\right\}$ is equi-integrable,

ii) $\nabla U_{k}(x) \in \Lambda_{\alpha} \cup \Lambda_{\beta}$, a.e. $x \in \Omega \forall k, \int_{\Omega} V\left(x, \nabla U_{k}(x)\right) d x=t_{0}, \forall k$

iii) $\lim _{k \rightarrow \infty} \int_{\Omega} W\left(x, \nabla U_{k}(x)\right) d x=\int_{\Omega} \int_{M^{3 \times 3}} W(x, A) d \nu_{x}(A) d x$

Proof. It is enough to generalize to the three-dimensional case the proof used in ([2]) for the two-dimensional case. This is a straightforward generalization.

\section{Constrained quasiconvexification}

We would like to compute explicitly the constrained quasionvexification defined as

$$
C Q W(x, F, t)=\inf \left\{\int_{M^{3 \times 3}} W(x, A) d \nu(A): \nu \in \mathcal{A}(F, t)\right\}
$$

where $\mathcal{A}(F, t)$ is given in (6). This constrained quasiconvexification can be expressed as

$$
\inf _{\nu}\left\{\int_{M^{3 \times 3}} W(x, A) d \nu(A): F=\int_{M^{3 \times 3}} A d \nu(A), \int_{M^{3 \times 3}} V(x, A) d \nu(A)=t\right\}
$$

with $\nu$ a homogeneous $H^{1}$-Young measure with $\operatorname{supp}(\nu) \subset \Lambda_{\alpha} \cup \Lambda_{\beta}$.

For $(F, t)$ (and $x$ ) fixed, we are going to calculate the value in $(7)$, i.e. $C Q W(x, F, t)$. The main difficulty here is that we don't know explicitly the set of the admissible measures, which we note as $\mathcal{A}$. The plan to follow will be similar to the two-dimensional case. The first step is to calculate the minimum over a greater class of probability measures $\mathcal{A}^{*} \supset \mathcal{A}$ where $\mathcal{A}^{*}$ is the set of all polyconvex measures. In this way we obtain a lower bound ( the (constrained) polyconvexification). Once this bound is computed, we search a measure over a narrower class of measures ( the laminates) which will tell us that the bound is attained, so that we will have in fact computed the exact value $C Q W(x, F, t)$.

The polyconvexification $C P W(x, F, t)$ can be computed through the following optimization problem

$$
\min _{\nu} \int_{M^{3 \times 3}} W(x, A) d \nu(A)
$$


subject to

$$
\begin{gathered}
\nu=t \nu_{\alpha}+(1-t) \nu_{\beta}, \text { commutes with all (some) minors } \\
\operatorname{supp}\left(\nu_{\gamma}\right) \subset \Lambda_{\gamma}, \gamma=\alpha, \beta, \\
F=t \int_{\Lambda_{\alpha}} A d \nu_{\alpha}(A)+(1-t) \int_{\Lambda_{\beta}} A d \nu_{\beta}(A) .
\end{gathered}
$$

It is clear that the integral constraints have been incorporated in the decomposition of the measure $\nu$. Let us first examine the constraints. We introduce the following variables

$$
S_{\gamma}=\int_{R^{3}}|\lambda|^{2} d \nu_{\gamma}^{(1)}(\lambda), \quad \text { with } \gamma=\alpha, \beta,
$$

where $\nu_{\gamma}^{(1)}$ is the probability measure resulting from the projection of $\nu_{\gamma}$ onto the first row. On the other hand, we put

$$
F_{\gamma}=\int_{\Lambda_{\gamma}} A d \nu_{\gamma}(A) \text { for } \gamma=\alpha, \beta
$$

From the fact that the measure $\nu=t \nu_{\alpha}+(1-t) \nu_{\beta}$ commutes with the determinant, it is clear that

$$
A \in \Lambda_{\gamma} \Rightarrow\left\{\begin{array}{l}
\gamma A^{(1)}-A^{(2)} \times A^{(3)}+G=0 \\
\operatorname{det} A=A^{(1)} \cdot\left(A^{(2)} \times A^{(3)}\right)
\end{array}\right\} \Rightarrow \operatorname{det} A=\gamma\left|A^{(1)}\right|^{2}+F^{(1)} \cdot G,
$$

thanks to the commutations with the minors. In particular, applied to the determinant, it leads to

$$
\operatorname{det} F=t \int_{\Lambda_{\alpha}} \operatorname{det} A d \nu_{\alpha}(A)+(1-t) \int_{\Lambda_{\beta}} \operatorname{det} A d \nu_{\beta}(A) .
$$

Keeping in mind (8) and (9), we have that

$$
\operatorname{det} F=t \alpha S_{\alpha}+(1-t) \beta S_{\beta}+F^{(1)} \cdot G .
$$

The components of $F^{(2)} \times F^{(3)}$ are the second order minors which have been computed using the second and third row of the matrix $F$, and again the commutation with $\nu$ yields

$$
\begin{array}{r}
F^{(2)} \times F^{(3)}=\int_{R^{3 \times 3}} A^{(2)} \times A^{(3)} d \nu(A)= \\
t \alpha \int A^{(1)} d \nu_{\alpha}+(1-t) \beta \int A^{(1)} d \nu_{\beta}+G=t \alpha F_{\alpha}^{(1)}+(1-t) \beta F_{\beta}^{(1)}+G .
\end{array}
$$

Moreover

$$
F=t F_{\alpha}+(1-t) F_{\beta} \Rightarrow F^{(1)}=t F_{\alpha}^{(1)}+(1-t) F_{\beta}^{(1)} .
$$


Using the last equalities, we can deduce

$$
\left\{\begin{array}{l}
F_{\alpha}^{(1)}=\frac{1}{t(\beta-\alpha)}\left(\beta F^{(1)}-F^{(2)} \times F^{(3)}+G\right) \\
F_{\beta}^{(1)}=\frac{-1}{(1-t)(\beta-\alpha)}\left(\alpha F^{(1)}-F^{(2)} \times F^{(3)}+G\right) .
\end{array}\right.
$$

On the other hand, by the Jensen's inequality

$$
S_{\gamma} \geq\left|\int_{R^{3}} \lambda d \nu_{\gamma}^{(1)}(\lambda)\right|^{2}=\left|F_{\gamma}^{(1)}\right|^{2}, \text { with } \gamma=\alpha, \beta,
$$

and bearing in mind (10), we can write

$$
\begin{aligned}
t^{2}(\beta-\alpha)^{2} S_{\alpha} \geq \beta^{2}\left|F^{(1)}\right|^{2}+\left|F^{(2)} \times F^{(3)}\right|^{2}+|G|^{2}- & \\
& 2 \beta \operatorname{det} F+2\left(\beta F^{(1)}-F^{(2)} \times F^{(3)}\right) \cdot G, \\
(1-t)^{2}(\beta-\alpha)^{2} S_{\beta} \geq \alpha^{2}\left|F^{(1)}\right|^{2}+ & \left|F^{(2)} \times F^{(3)}\right|^{2}+|G|^{2}- \\
& 2 \alpha \operatorname{det} F+2\left(\alpha F^{(1)}-F^{(2)} \times F^{(3)}\right) \cdot G .
\end{aligned}
$$

The cost functional can be rewriten in terms of the $S_{\gamma}$ variables as follows

$$
t a_{\alpha} S_{\alpha}+(1-t) a_{\beta} S_{\beta}
$$

Hence, we can rewrite the original optimization problem as a mathematical programming problem

$$
\underset{\left(S_{\alpha}, S_{\beta}\right)}{\operatorname{minimize}} t a_{\alpha} S_{\alpha}+(1-t) a_{\beta} S_{\beta}
$$

subject to

$$
\operatorname{det} F=t \alpha S_{\alpha}+(1-t) \beta S_{\beta}
$$

$$
\begin{aligned}
& \begin{aligned}
\beta^{2}\left|F^{(1)}\right|^{2}+\left|F^{(2)} \times F^{(3)}\right|^{2}+|G|^{2}-2 \beta \operatorname{det} F+ \\
2\left(\beta F^{(1)}-F^{(2)} \times F^{(3)}\right) \cdot G-t^{2}(\beta-\alpha)^{2} S_{\alpha} \leq 0, \\
\alpha^{2}\left|F^{(1)}\right|^{2}+\left|F^{(2)} \times F^{(3)}\right|^{2}+|G|^{2}-2 \alpha \operatorname{det} F+ \\
2\left(\alpha F^{(1)}-F^{(2)} \times F^{(3)}\right) \cdot G-(1-t)^{2}(\beta-\alpha)^{2} S_{\beta} \leq 0,
\end{aligned}
\end{aligned}
$$

where the parameters $\alpha, \beta, a_{\alpha}, a_{\beta}$ are part of the data set of the original problem, and the variables $t, F$ (and $x$ ) are fixed.

The first issue about this mathematical programming problem is to compute the admissible set for the variables $\left(S_{\alpha}, S_{\beta}\right)$. This is determined by the intersection of two semi-planes and one line; therefore the admissible set will 
Figure 1: Mathematical programming problem

be the segment of the line within the two semi-planes. This is easy to see geometrically in Figure 1.

The admissible set will be non-empty when the point of intersection where the two inequality constraints become equalities $\left(P_{0}\right.$ in Figure 1$)$ is under the line represented by the equality constraint. This amounts to

$$
\begin{array}{r}
\alpha \beta(t \alpha+(1-t) \beta)\left|F^{(1)}\right|^{2}+ \\
((1-t) \alpha+t \beta)\left(\left|F^{(2)} \times F^{(3)}\right|^{2}+|G|^{2}-2\left(F^{(2)} \times F^{(3)}\right) \cdot G\right) \\
+\left(2 \alpha \beta+t(1-t)(\beta-\alpha)^{2}\right)\left(F^{(1)} \cdot G-\operatorname{det} F\right) \leq 0 .
\end{array}
$$

The second issue is to decide the point(s) where the minimum value is attained. It is clear that the optimal point depends of the coefficients $a_{\alpha}, a_{\beta}$. We have previously determined that the admissible set (when it is non-empty) is a segment, and the functional cost is a linear functional. Therefore the minimum value will be attained at one of the extreme points of the segment, or become constant over all of the segment, depending on the particular values of the parameters $a_{\alpha}, a_{\beta}$. Let

$$
h(x)=\beta a_{\alpha}(x)-\alpha a_{\beta}(x)
$$

It is easy to compute that the minimum value, which depends on the sign of the function $h(x)$, therefore assuming that (11) holds, 


$$
\begin{array}{r}
C P W(x, t, F)=\frac{h(x)}{t \beta(\beta-\alpha)^{2}}\left(\beta^{2}\left|F^{(1)}\right|^{2}+\left|F^{(2)} \times F^{(3)}\right|^{2}+|G|^{2}\right. \\
\left.-2 \beta \operatorname{det} F+2\left(\beta F^{(1)}-F^{(2)} \times F^{(3)}\right) \cdot G\right)+\frac{a_{\beta}(x)}{\beta}\left(\operatorname{det} F-G \cdot F^{(1)}\right) \\
\text { if } h(x) \geq 0, \\
C P W(x, t, F)=\frac{-h(x)}{(1-t) \alpha(\beta-\alpha)^{2}}\left(\alpha^{2}\left|F^{(1)}\right|^{2}+\left|F^{(2)} \times F^{(3)}\right|^{2}+|G|^{2}\right. \\
\left.-2 \alpha \operatorname{det} F+2\left(\alpha F^{(1)}-F^{(2)} \times F^{(3)}\right) \cdot G\right)+\frac{a_{\alpha}(x)}{\alpha}\left(\operatorname{det} F-G \cdot F^{(1)}\right), \\
\text { if } h(x) \leq 0 .
\end{array}
$$

This lower bound will become an exact value for $C Q W(x, t, F)$ if these extreme points can be attained as the second moments of some measures $\nu_{\alpha}, \nu_{\beta}$ (according to (8)) and that the convex combination $t \nu_{\alpha}+(1-t) \nu_{\beta}$ is a laminate.

We explicitly find such measures in the case $h(x) \geq 0$, where the extreme point is attained when

$$
\begin{array}{r}
\beta^{2}\left|F^{(1)}\right|^{2}+\left|F^{(2)} \times F^{(3)}\right|^{2}+|G|^{2} \\
-2 \beta \operatorname{det} F+2\left(\beta F^{(1)}-F^{(2)} \times F^{(3)}\right) \cdot G-t^{2}(\beta-\alpha)^{2} S_{\alpha}=0 .
\end{array}
$$

In this case we have

$$
S_{\alpha}=\int_{R^{3}}\left|A^{(1)}\right|^{2} d \nu_{\alpha}(A)=\left|F_{\alpha}^{(1)}\right|^{2},
$$

and bearing in mind that the functional $\int_{\Lambda_{\alpha}}\left|A^{(1)}\right|^{2} d \nu_{\alpha}(A)$ is strictly convex, we can deduce that $\nu_{\alpha}=\delta_{G_{\alpha}}$ for $G_{\alpha} \in \Lambda_{\alpha}$ and $G_{\alpha}^{(1)}=F_{\alpha}^{(1)}$.

Let the functions $g, h: \mathbb{R}^{3 \times 3} \rightarrow \mathbb{R}$ be defined as

$$
\begin{gathered}
g(F)=\alpha^{2} \beta^{2}\left|F^{(1)}\right|^{4}+\left|F^{(2)} \times F^{(3)}\right|^{4}+\left(\alpha^{2}+6 \alpha \beta+\beta^{2}\right)\left(\operatorname{det} F-G \cdot F^{(1)}\right)^{2} \\
-2 \alpha \beta\left|F^{(1)}\right|^{2}\left|F^{(2)} \times F^{(3)}-G \cdot F^{(1)}\right|^{2}-2 \alpha \beta(\alpha+\beta)\left|F^{(1)}\right|^{2}\left(\operatorname{det} F-G \cdot F^{(1)}\right) \\
-2(\alpha+\beta)\left|F^{(2)} \times F^{(3)}-G \cdot F^{(1)}\right|^{2}\left(\operatorname{det} F-G \cdot F^{(1)}\right), \\
h(F)=(\alpha+\beta)\left(\operatorname{det} F-G \cdot F^{(1)}\right)-\alpha \beta\left|F^{(1)}\right|^{2}-\left|F^{(2)} \times F^{(3)}-G\right|^{2} .
\end{gathered}
$$

Lemma 1 Let $F \notin \Lambda_{\alpha} \cup \Lambda_{\beta}$ and such that $g(F) \geq 0$ and $h(F) \geq 0$. Then, there exist $a \in \mathbb{R}^{3},(r, s) \in \mathbb{R}^{2}$ and $\lambda \in \mathbb{R}^{+}$such that,

$$
F+\left(\begin{array}{c}
a \\
r a \\
s a
\end{array}\right) \in \Lambda_{\alpha}, F-\lambda\left(\begin{array}{c}
a \\
r a \\
s a
\end{array}\right) \in \Lambda_{\beta} .
$$


Proof. Conditions (12) can be written as

$$
\left\{\begin{array}{l}
\alpha a-s F^{(2)} \times a-r a \times F^{(3)}=-\left(\alpha F^{(1)}-F^{(2)} \times F^{(3)}+G\right), \\
\beta a-s F^{(2)} \times a-r a \times F^{(3)}=\frac{1}{\lambda}\left(\beta F^{(1)}-F^{(2)} \times F^{(3)}+G\right),
\end{array}\right.
$$

where we have

$$
a=\frac{1}{(\beta-\alpha)}\left[\left(\alpha F^{(1)}-F^{(2)} \times F^{(3)}+G\right)+\frac{1}{\lambda}\left(\beta F^{(1)}-F^{(2)} \times F^{(3)}+G\right)\right] .
$$

Thus the above system has solutions if and only if

$$
a \cdot\left(\alpha F^{(1)}-F^{(2)} \times F^{(3)}+G+\alpha a\right)=0 .
$$

The necessity is elementary while for the sufficiency simply notice that any vector $\left(\alpha F^{(1)}-F^{(2)} \times F^{(3)}+G+\alpha a\right)$ orthogonal to $a$, can be decomposed as a linear combination of the basis $\left\{F^{(2)} \times a, a \times F^{(3)}\right\}$.

It is elementary to check that (14) is equivalent to $S_{F}(1 / \lambda)=0$ with

$$
\begin{array}{r}
S_{F}(x)=x^{2} \beta\left|\alpha F^{(1)}-F^{(2)} \times F^{(3)}+G\right|^{2} \\
+x(\alpha+\beta)\left(\alpha F^{(1)}-F^{(2)} \times F^{(3)}+G\right) \cdot\left(\beta F^{(1)}-F^{(2)} \times F^{(3)}+G\right) \\
+\alpha\left|\beta F^{(1)}-F^{(2)} \times F^{(3)}+G\right|^{2} .
\end{array}
$$

$S_{F}$ is a second degree polynomial that will have real roots if its discriminant is non negative, i.e., $g(F) \geq 0$. On the other hand, it is easy to check that $S_{F}(0)>0$ and therefore there will exist positive solutions if $S_{F}$ is decreasing in 0 , i.e., $h(F) \geq 0$. It is easy to check that these conditions are the hypotheses of the lemma.

There exist then two solutions, namely

$$
\begin{array}{r}
\frac{1}{\lambda_{i}}=\frac{1}{2\left(\beta \alpha^{2}\left|F^{(1)}\right|^{2}+\beta\left|F^{(2)} \times F^{(3)}-G\right|^{2}-\alpha\left(\operatorname{det} F-G \cdot F^{(1)}\right)\right)} \\
\left((\beta+\alpha)\left((\alpha+\beta)\left(\operatorname{det} F-G \cdot F^{(1)}\right)\right)-\alpha \beta\left|F^{(1)}\right|^{2}+\left|F^{(2)} \times F^{(3)}-G\right|^{2}\right) \\
\left.+(-1)^{i}(\beta-\alpha) \sqrt{g(F)}\right)
\end{array}
$$

and the corresponding $\left(r_{i}, s_{i}\right)$ and $a_{i}$ with $i=1,2$.

Let us put $P_{F}(t)$ to designate

$$
\begin{array}{r}
(\beta-\alpha)^{2}\left(\operatorname{det} F-G \cdot F^{(1)}\right) t^{2}+\left(\beta\left|\alpha F^{(1)}-F^{(2)} \times F^{(3)}+G\right|^{2}\right. \\
\left.\left.-\alpha\left|\beta F^{(1)}-F^{(2)} \times F^{(3)}+G\right|^{2}-(\beta-\alpha)^{2}\left(\operatorname{det} F-G \cdot F^{(1)}\right)\right)\right) t \\
+\alpha\left|\beta F^{(1)}-F^{(2)} \times F^{(3)}+G\right|^{2} .
\end{array}
$$


After some additional algebraic manipulations, one can show that condition (11) is equivalent to $P_{F}(t) \leq 0$. Moreover, it is elementary to check that $P_{F}(t)=S_{F}\left(\frac{t}{1-t}\right)$, and therefore the conditions which guarantee that $t \in$ $(0,1)$ are $g(F) \geq 0$ and $h(F) \geq 0$. Let us put $t_{i}, i=1,2$ the two roots of $P_{F}$

$$
\begin{array}{r}
t_{i}=\frac{1}{2}+\frac{1}{2(\beta-\alpha)(\operatorname{det} F)-G \cdot F^{(1)}}\left[\alpha \beta\left|F^{(1)}\right|^{2}-\left|F^{(2)} \times F^{(3)}-G\right|^{2}\right. \\
\left.+(-1)^{i} \sqrt{g}(F)\right] i=1,2 .
\end{array}
$$

It is clear that these remarks imply that the set where $C P W$ is finite can be described as the pairs $(t, F)$ such that

$$
g(F) \geq 0, h(F) \geq 0, t \in\left[t_{1}, t_{2}\right] .
$$

To summarize, we have that for a pair $(t, F)$ verifying (15), by Lemma 1 , we can guarantee that there exist exactly two first-order laminates supported in $\Lambda_{\alpha} \cup \Lambda_{\beta}$ and barycenter $F$; from here we can obtain a second-order laminate which attains the optimal value of $C P W$. We can see the geometrical situation in Figure 2.

Figure 2: Spatial situation

We are now going to work in the plane determined by $F$ and the two rank-ones directions. We seek a matrix $M_{\alpha}$ such that,

$$
\begin{gathered}
M_{\alpha} \in \Lambda_{\alpha} \\
M_{\alpha}^{(1)}=\frac{1}{t(\beta-\alpha)}\left(\beta F^{(1)}-F^{(2)} \times F^{(3)}\right) .
\end{gathered}
$$


A matrix $M$ belongs to that plane if

$$
M=F+\sigma A_{1}+\mu A_{2}
$$

where

$$
A_{i}=\left(\begin{array}{c}
a_{i} \\
r_{i} a_{i} \\
s_{i} a_{i}
\end{array}\right) i=1,2,
$$

are the rank-one directions determined in Lemma 1 and $(\sigma, \mu) \in R^{2}$ are arbitrary.

When we impose to a matrix in the plane (17) that its first row be given by (16), we find a unique pair $\left(\sigma^{*}, \mu^{*}\right)$ given by

$$
\begin{aligned}
\sigma^{*} & =\frac{\lambda_{1}\left(t+\lambda_{2} t-\lambda_{2}\right)}{t\left(\lambda_{1}-\lambda_{2}\right)}, \\
\mu^{*} & =\frac{-\lambda_{2}\left(t+\lambda_{1} t-\lambda_{1}\right)}{t\left(\lambda_{1}-\lambda_{2}\right)} .
\end{aligned}
$$

Note that $\mu^{*}=1-\sigma^{*}$. Then the issue is to check if $M_{\alpha}=F+\sigma^{*} A_{1}+\mu^{*} A_{2}$ belongs to $\Lambda_{\alpha}$.

We take a matrix $M$ in the plane (17) and force that $M$ to belong to $\Lambda_{\alpha}$. Having in mind (13),

$$
\begin{aligned}
& s_{i} F^{(2)} \times a_{i}+r_{i} a_{i} \times F^{(3)}=\alpha F^{(1)}-F^{(2)} \times F^{(3)}+G+\alpha a_{i} \text { with } i=1,2, \\
& \left.\begin{array}{l}
M=F+\sigma A_{1}+\mu A_{2} \\
M \in \Lambda_{\alpha}
\end{array}\right\} \Leftrightarrow
\end{aligned}
$$

$\alpha\left(F^{(1)}+\sigma a_{1}+\mu a_{2}\right)+G=$

$\left(F^{(2)}+\sigma r_{1} a_{1}+\mu r_{2} a_{2}\right) \times\left(F^{(3)}+\sigma s_{1} a_{1}+\mu s_{2} a_{2}\right)=$

$F^{(2)} \times F^{(3)}+\sigma\left(s_{1} F^{(2)} \times a_{1}+r_{1} a_{1} \times F^{(3)}\right)+\mu\left(s_{2} F^{(2)} \times a_{2}+r_{2} a_{2} \times F^{(3)}\right)=$ \{using (13)\}

$F^{(2)} \times F^{(3)}+(\sigma+\mu)\left(\alpha F^{(1)}-F^{(2)} \times F^{(3)}+G\right)+\alpha\left(\sigma a_{1}+\mu a_{2}\right) \Leftrightarrow$ $(1-\sigma-\mu)\left(\alpha F^{(1)}-F^{(2)} \times F^{(3)}+G\right)=0$.

Then a matrix $M=F+\sigma A_{1}+\mu A_{2}$ belongs to $\Lambda_{\alpha}$, if and only if $\mu=$ $1-\sigma$. From this we can deduce that the intersection between $\Lambda_{\alpha}$ and the plane determined by the rank-one directions is a linear manifold, and most important, $M_{\alpha}=F+\sigma^{*} A_{1}+\mu^{*} A_{2}$ belongs to $\Lambda_{\alpha}$, since $\mu^{*}=1-\sigma^{*}$. Therefore, the characterization of this intersection can be written in the form 


$$
M=F+\sigma A_{1}+(1-\sigma) A_{2}=\sigma F_{\alpha, 1}+(1-\sigma) F_{\alpha, 2},
$$

where $F_{\alpha, i}=F+A_{i}, i=1,2$, are the intersection between the rank-one directions and the $\Lambda_{\alpha}$ manifold (the black points in Figure 2).

In a similar way, we can show that the intersection between the rank-one directions and the $\Lambda_{\beta}$ manifold is again another linear manifold determined by

$$
M=F+\sigma A_{1}+(1-\sigma) A_{2}=\sigma F_{\beta, 1}+(1-\sigma) F_{\beta, 2}
$$

where $F_{\beta, i}=F+\lambda_{i} A_{i}, i=1,2$, are the intersection between the rank-one directions and the $\Lambda_{\beta}$ manifold (the green points in Figure 2).

Then we have $\nu_{\alpha}=\delta_{M_{\alpha}}$ such that $\operatorname{supp}\left(\nu_{\alpha}\right) \subset \Lambda_{\alpha}$. If we seek $\nu_{\beta}$ as a convex combination of two Dirac masses with support in the $\Lambda_{\beta}$ manifold, we may produce a second-order laminate supported in $\Lambda_{\alpha} \cup \Lambda_{\beta}$. The situation in the plane can be drawn as in Figure 3 where laminates are shown with green and yellow colors.

Figure 3: Plane of rank-one directions

Let $\bar{F}_{\beta, i}=M_{\alpha}+l_{i}\left(F_{\beta, i}-F_{\alpha, i}\right)$. It is easy to check that

$$
\bar{F}_{\beta, i}=l_{i}^{*} F_{\beta, 1}+\left(1-l_{i}^{*}\right) F_{\beta, 2}
$$

with

$$
l_{i}^{*}=\frac{\lambda_{i}-t\left(\lambda_{2}+1\right)}{t\left(\lambda_{1}-\lambda_{2}\right)} .
$$


To sum up, if we consider the matrices,

$$
\begin{array}{ccc}
F_{\alpha, i}=F+A_{i}, F_{\beta, i}=F-\lambda_{i} A_{i} \quad \text { with } & A_{i}=\left(\begin{array}{c}
a_{i} \\
r_{i} a_{i} \\
s_{i} a_{i}
\end{array}\right), i=1,2, \\
M_{\alpha}=\sigma^{*} F_{\alpha, 1}+\left(1-\sigma^{*}\right) F_{\alpha, 2} \quad \text { with } \quad \sigma^{*}=\frac{\lambda_{1}\left(t+\lambda_{2} t-\lambda_{2}\right)}{t\left(\lambda_{1}-\lambda_{2}\right)}, \\
\bar{F}_{\beta, i}=l_{i}^{*} F_{\beta, 1}+\left(1-l_{i}^{*}\right) F_{\beta, 2} \quad \text { with } \quad l_{i}^{*}=\frac{\lambda_{i}-t\left(\lambda_{2}+1\right)}{t\left(\lambda_{1}-\lambda_{2}\right)},
\end{array}
$$

and the scalars

$$
\rho_{i, j}=\frac{t-\lambda_{i}(1-t)}{\lambda_{j}-\lambda_{i}} \quad \tau_{i, j}=\frac{t-\lambda_{j}(1-t)}{t\left(\lambda_{i}+1\right)-\lambda_{j}},
$$

we can define the second-order laminate with support on $\Lambda_{\alpha} \cup \Lambda_{\beta}$, barycenter $F$, and mass in $\Lambda_{\alpha}$ equal to $t$, by putting

$$
\nu_{i, j}=\tau_{i, j} \delta_{F_{\beta, i}}+\left(1-\tau_{i, j}\right)\left(\rho_{i, j} \delta_{\bar{F}_{\beta, j}}+\left(1-\rho_{i, j}\right) \delta_{M_{\alpha}}\right)
$$

with $i, j \in\{1,2\}, i \neq j$ where,

$$
\operatorname{det}\left(F_{\beta, j}-M_{\alpha}\right)=0
$$

and

$$
\operatorname{det}\left(F_{\beta, i}-\left(\rho_{i, j} \bar{F}_{\beta, j}+\left(1-\rho_{i, j}\right) M_{\alpha}\right)\right)=0 .
$$

A similar result holds for the other point where the optimal value is attained.

This finishes the computation of $C Q W(x, t, F)$ and the proof of Theorem 1.

\section{References}

[1] Allaire, G., Shape optimization by the homogenization method, Springer, 2002 .

[2] Aranda, E. and Pedregal P., Constrained envelope for a general class of design problems, Disc. Cont. Dyn. Syst., Supplement Volume, (2003), $30-41$.

[3] Bellido, J.C., Explicit computation of the relaxed density coming from a three-dimensional optimal design problem, Nonlinear Analysis $\mathbf{5 2}$ (2003), 1709-1726.

[4] Chipot, M., Elements of Nolinear Analysis, Birkhäuser, Basel, 2000.

[5] Dacorogna, B., Direct method in the calculus of variations, Springer, 1989.

[6] Ekeland, I. and Téman, R., Convex Analisys and Variational Problems SIAM, Philadelphia, 1999. 
[7] Graham, C. R. and Henyey, F. S., clebsch representation near points where the vorticity vanishes, Physis of Fluids, 12, no 4, (2000), 744-746.

[8] Kotiuga, P.R., Clebsch potentials and the visualization of threedimensional solenoidal vector fields, IEEE Trans. Magnetics, 27, no. 5, (1991), 3986-3989

[9] Lipton, R. and Velo, A.P., Optimal design of gradient field with applications to electrostatics, in Nonlinear Partial Differential Equations and Their Applications, College de France Seminar Volume XIV, (D. Cioranescu and J.L. Lions eds.), 2002

[10] Pedregal, P. Vector variational problems and applications to optimal design, ESAIM-COCV (to appear).

[11] Nigam, S.D., Usha R. and Swaminathan, k., Divergence-free vectors fields, Jour. Math. Phy. Sci. 14, no. 5 (1980), 523-527

[12] Pedregal, P. Constrained quasiconvexification of the square of the gradient of the state in optimal design, Quart. Appl. Math., 62 , no 3 (2004), 459-470.

[13] Pedregal, P. Optimal design in 2-D conductivity for quadratic functionals in the field, Prc. Advanced NATO Conference in Non-linear homogenization, Varsovia, 2004

[14] Pedregal, P. Parametrized Measure and Variationales Principles, Birkhäuser, Basel, 1997.

[15] Shivamoggi, B.K., Theorical Fluid Dynamics, John Wiley and Sons, Inc., 1998

[16] Tartar, L. Remarks on optimal design problems, in Calculus of Variations, Homogenizacion and Continuum Mechasnics, (G. Buttazzo, G. Bouchitte and P. Suquet, eds.), World Scientific, Singapore, (1994) 279296.

[17] Tartar, L. An introduction to the homogenization method in optimal design, Springer Lecture Notes in Math. ,1740, (2000) 47-156.

[18] Yih, C.S., Stream functions in three-dimensional flows, La Houille Blanche no 3 (1957) 445-450. 